\title{
History-dependent changes of Gamma distribution in multistable
}

2

\section{perception}

Short title: History-dependent changes of Gamma distribution in multistability

\author{
Alexander Pastukhov ${ }^{1,2^{\star}}$, Malin Styrnal ${ }^{1}$, Claus-Christian Carbon ${ }^{1,2}$
}

${ }^{1}$ Department of General Psychology and Methodology, University of Bamberg, Bamberg, Bavaria, Germany.

${ }^{2}$ Forschungsgruppe EPAEG (Ergonomics, Psychological ÆEsthetics, Gestalt), Bamberg, Bavaria, Germany.

*Correspondence to pastukhov.alexander@gmail.com.

\section{Abstract}

Multistable perception - spontaneous switches of perception when viewing a stimulus compatible with several distinct interpretations - is often characterized by the distribution of durations of individual dominance phases. For continuous viewing conditions, these distributions look remarkably similar for various multistable displays and are typically described using Gamma distribution. Moreover, durations of individual dominance phases show a subtle but consistent dependence on prior perceptual experience with longer dominance phases tending to increase the duration of the following ones, whereas the shorter dominance leads to similarly shorter durations. One way to generate similar switching behavior in a model is by using a combination of cross-inhibition, self-adaptation, and neural noise with multiple useful models being built on this principle. Here, we take a closer look at the history-dependent changes in the distribution of durations of dominance phases. Specifically, we used Gamma distribution and allowed both its parameters - shape and scale - to be linearly dependent on the prior perceptual experience at two timescales. We fit a hierarchical Bayesian model to five datasets that included binocular rivalry, 
Necker cube, and kinetic-depth effects displays, as well as data on binocular rivalry in children and on binocular rivalry with modulated contrast. For all datasets, we found a consistent change of the distribution shape with higher levels of perceptual history, which can be viewed as a proxy for perceptual adaptation, leading to a more normal-like shape of the Gamma distribution. When comparing real observers to matched simulated dominance phases generated by a spiking neural model of bistability, we found that although it matched the positive history-dependent shift in the shape parameter, it also predicted a negative change of scale parameter that did not match empirical data. We argue that our novel analysis method, the implementation is available freely at the online repository, provides additional constraints for computational models of multistability.

\section{Author Summary}

Multistable perception occurs when one continuously views a figure that can be seen in two distinct ways. A classic old-young woman painting, a face-vase figure, or a Necker cube are examples easy to find online. The endless spontaneous switches of your perception between the alternatives inform us about the interplay of various forces that shape it. One way to characterize these switches is by looking at their timing: How long was a particular image dominant, how did that reflect what you have seen previously, the focus of your attention, or the version of the figure that we showed you? This knowledge allows us to build models of perception and test them against the data we collected. As models grow more elaborate, we need to make tests more elaborate as well and for this, we require more precise and specific ways to characterize your perception. Here, we demonstrate how your recent perceptual experience - which of the alternative images did you see and for how long - predicts subtle but consistent changes in the shape of the distribution that describes perceptual switching. We believe it to be a more stringent test by demonstrating how a classical model of bistability fails on it. 


\section{Keywords}

Multistable perception; bistable perception; autocorrelation; gamma distribution; serial dependence; history dependence; binocular rivalry; Necker cube; kinetic-depth effect; Bayesian

\section{Introduction}

We rely on unambiguous perception to be able to initiate actions in a very fast and clearly directed way. The real challenge of perception is to generate such a clear and single representation of the outside world that is based on inherently noisy and ambiguous sensory information. As the mental act of creating such perception must evidently be based on perceptual models, the representation will not always match the actual state of the external world leading to characteristic and highly informative visual illusions [1]. Sometimes, sensory information is compatible with several comparably likely perceptual interpretations [2]. A few of these so-called multistable figures can be seen in Figure 1A-C, and many more stimuli do exist and are systematically investigated, including an auditory [3], tactile [4], and even olfactory bistable stimuli [5]. Thus, multistable perception is particularly interesting because it is a general phenomenon, so understanding it advances our knowledge about the architecture of our perceptual system and perceptual decision making.

A

B
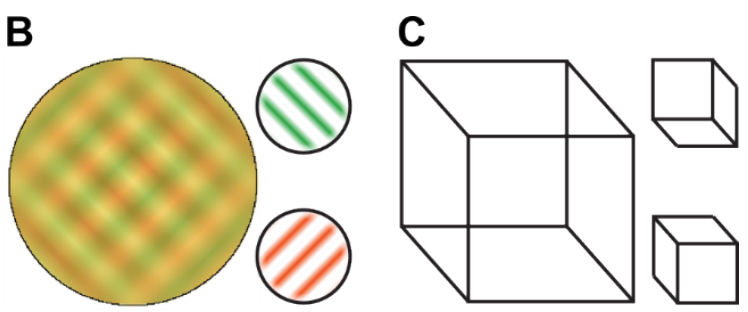

D

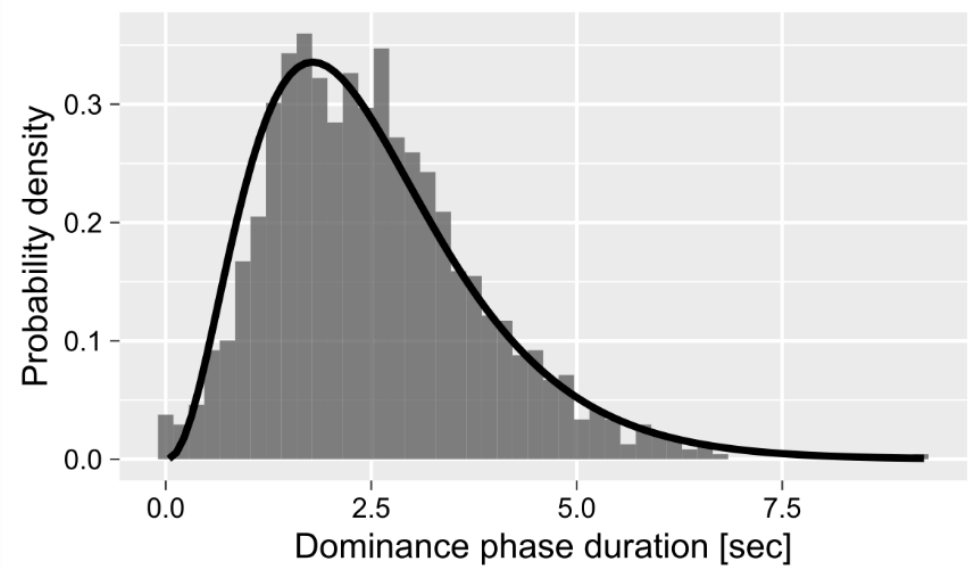


Figure 1. Bistable displays (A, B, C) and a typical distribution of dominance phase durations (D). A) In the kinetic-depth effect, a 2D onscreen-motion produces an alternating perception of a rotating object. B) Binocular rivalry produces alternating dominance of two orthogonal gratings. C) Necker cube can be perceived in two orientations. D) Distribution of dominance phases for a participant, who viewed kinetic-depth effect displays, with an overlaid fitted gamma distribution.

When multistable displays are presented continuously, their perception is often characterized by a distribution of duration times or, conversely, by the alternation rate. Although duration times vary strongly between subjects, the shape of the distributions is remarkably similar [6], see Figure 1D.

This consistency is viewed as a "hallmark" of multistable perception [7] and is frequently used as a first check when characterizing a new multistable display [8]. There is a debate about which theoretical distribution fits the empirical dominance phase durations the best with many suggestions that included gamma, exponential, Weibull, normal, Capocelli-Ricciardi, beta rate, exgaussian, and log-normal distributions [9-15]. Among those, the gamma distribution is generally considered to be the canonical distribution for fitting the data $[9,16,17]$. Its specific appeal is that it allows one to conceptualize perceptual alternations as a Poisson process that occurs after $\alpha$ discrete stochastic events, which occurr independently [9,17]. Interestingly, this conceptualization predicts that the shape parameter must be an integer number but the evidence for this is not conclusive [16].

Another important property of dominance phase durtions is a subtle but consistent dependence of their duration on prior perceptual history. This dependence is particularly evident when an unambiguous display precedes the bistable one. Here, an artificially prolonged dominance of a particular percept leads to longer dominance of the alternative percept during a consequent continuous presentation $[18,19]$. Although less pronounced, the same serial dependence is observed when fully ambiguous bistable displays are viewed continuously. A typical approach is to compute an autocorrelation of dominance phase durations with different lags [17] and a typical 
result is a small (0.1-0.2) but significant and consistently positive autocorrelation for lag 1 [20]. In other words, longer dominance phases tend to be followed by similarly long ones and, conversely, short phases tend to be followed by comparably short ones. Alternatively, prior perceptual history can be quantified using a leaky integrator with a fitted time constant [21]. The latter approach tends to produce a higher correlation between preceding history and the following dominance phase. Thus, it is likely to be a more accurate estimate of the perceptual history and, therefore, was used in the current study.

Typical models of bistable perception assume that it is borne out as an interplay between crossinhibition, self-adaptation, and noise [22-25]. The latter appears to be the main driver of perceptual alternations [26], whereas the former ensures perceptual exclusivity. The dependence of dominance phase duration's on prior perceptual history described above is thought to reflect noisy self-adaptation, so that high levels of adaptation of a particular percept allow for prolonged dominance of the alternative [20]. Conversely, similarly low levels of adaptation minimize its effect, so that perceptual alterations are driven primarily by stochastic factors [21,27].

As summarized above, the prior work showed that the mean of distribution of dominance durations is consistently shifted due to changes in adaptation levels. However, for the gamma distribution, a particular change of the mean is consistent with various combinations of changes to its shape and scale parameters (Figure 2). For example, a higher contrast in binocular rivalry displays results in shorter mean dominance phase durations, which is a product of a simultaneous increase in the shape and decrease in the scale parameters [20]. This change is more evident when the balance between adaptation and noise is manipulated by reducing the former, leading to an exponential distribution, e.g., gamma distribution with shape=1 [27]. Similarly, modelling demonstrates a consistent change in the gamma distribution when the balance between noise and adaptation is manipulated ranging from exponential, when dominated by noise, to normal, when dominated by adaptation [25]. Thus, the parameters of the gamma distribution should also reflect 

noise.

A

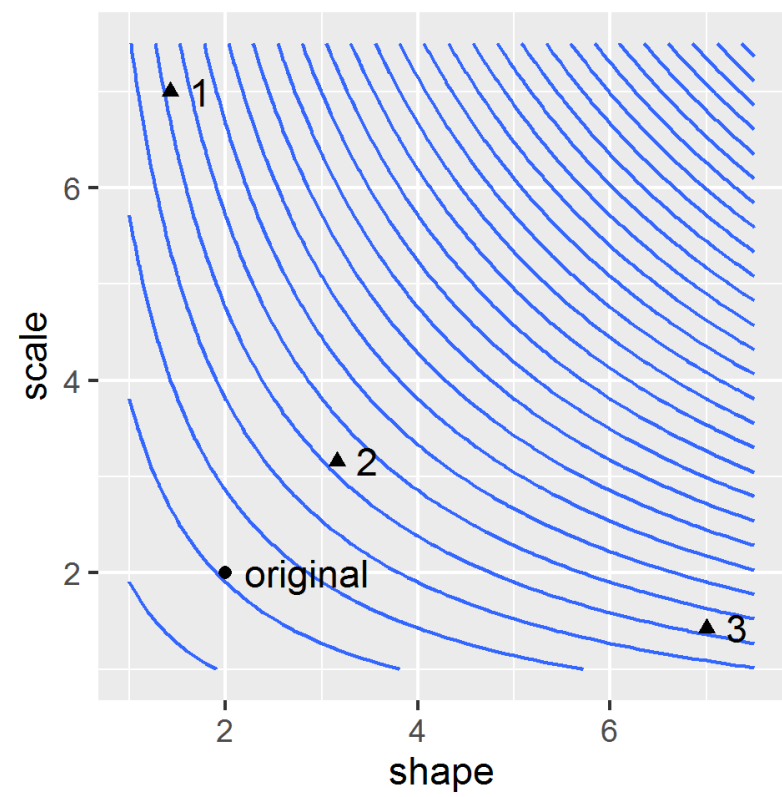

115
B

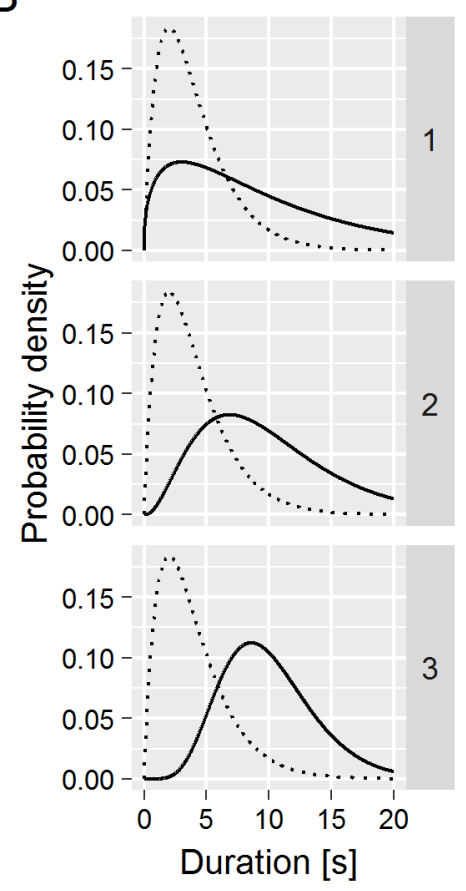

Figure 2. The same change in the mean of the gamma distribution can be implemented as various combinations of changes to its shape and scale parameters. A) Isolines indicate parameters'

combinations that produce the same mean. B) Three example distributions that have the same higher mean as compared to the original (dashed line).

In the present study, we sought to characterize the changes to gamma distribution using prior perceptual history computed via leaky integrator [21]. Specifically, we computed perceptual history for two timescales - short $\left(\tau=0.9<T_{\text {dom }}>\right.$, where $<T_{\text {dom }}>$ stands for mean dominance duration) and long $\left(\tau=30<T_{\text {dom }}>\right)$. Then, we used a hierarchical Bayesian model with gamma distribution allowing both parameters to vary with respect to perceptual history, stimulus contrast, and age of participants. We demonstrate that higher levels of perceptual history are associated with positive changes in shape parameter, leading to more "Gaussian-like" distribution, but we found no consistent changes in scale parameter. Finally, we fitted the same model on data 
simulated via a spiking neural model of bistability [22]. We observed that these did not fully match empirical observations, as positive changes in shape were accompanied by equally strong, and absent for empirical data, negative changes in the scale parameter. We conclude that our novel method improves our ability to characterize time-series of perceptual alternations and provides additional constraints for their models.

\section{Materials and Methods}

\section{Data sets}

We used two previously published data sets together with newly measured data (see Table $\mathbf{1}$ and the description below). The first data set, reported in [28], contains results for binocular rivalry (BR), kinetic-depth effect (KD), and Necker cube (NC) displays measured in an adult population. Second, reported in [29] used BR display measured in three age groups (9, 12, and 21 years old). For details please refer to the corresponding papers. We have included [28] and the new data set in the open data repository. However, the data set that contained children was not included as we had no permission to disclose it.

Table 1. Summary of data sets used in the study. BR - binocular rivalry, KD - kinetic depth effect display, NC- Necker cube.

\begin{tabular}{|c|c|c|c|c|c|c|}
\hline Source & $\begin{array}{l}\text { Dataset } \\
\text { Name }\end{array}$ & Display & Contrast & Age & $\begin{array}{l}\text { Number of } \\
\text { participants }\end{array}$ & $\begin{array}{l}\text { Number of } \\
\text { dominance } \\
\text { phases }\end{array}$ \\
\hline \multirow{3}{*}{$\begin{array}{l}\text { (Pastukhov et } \\
\text { al., 2013b) }\end{array}$} & $\mathrm{BR}$ & $\mathrm{BR}$ & $50 \%$ & & 8 & 6,698 \\
\hline & $K D$ & $K D$ & - & & 11 & 43,456 \\
\hline & NC & NC & & & 5 & 3,940 \\
\hline \multirow{2}{*}{$\begin{array}{l}\text { (Hudak et al., } \\
\text { 2011) }\end{array}$} & \multirow{2}{*}{ Development } & \multirow{2}{*}{ BR } & \multirow{2}{*}{$50 \%$} & 9 & 19 & 12,769 \\
\hline & & & & 12 & 23 & 19,271 \\
\hline
\end{tabular}




\begin{tabular}{|c|c|c|c|c|c|c|}
\hline & & & & 21 & 16 & 13,284 \\
\hline \multirow{5}{*}{ New data } & \multirow{5}{*}{ Contrasts } & \multirow{5}{*}{ BR } & $6.25 \%$ & & \multirow{5}{*}{6} & 930 \\
\hline & & & $12.5 \%$ & & & 980 \\
\hline & & & $25 \%$ & & & 1,000 \\
\hline & & & $50 \%$ & & & 1,258 \\
\hline & & & $100 \%$ & & & 1,296 \\
\hline \multicolumn{6}{|c|}{ Total number of dominance phases } & 104,882 \\
\hline
\end{tabular}

For the new data set, we used binocular rivalry display with a procedure similar to that used in [28]

but with five contrasts levels $(6.25 \%, 12.5 \%, 25 \%, 50 \%, 100 \%)$. Binocular rivalry stimulus consisted

of two orthogonally oriented gratings (radius $0.9^{\circ}$, spatial frequency 2 cycles $/{ }^{\circ}$, one was tilted by

$45^{\circ}$ clockwise and one $45^{\circ}$ counter-clockwise). Participants viewed the display through a custom-

made mirror stereoscope ( $75 \mathrm{~cm}$ eye-screen distance) and reported on the eye dominance using a

keyboard, continuously pressing left when the counter-clockwise oriented grating was dominant,

indicated mixed phases. Each presentation lasted for two minutes. An experimental session

randomized, so that the five contrast conditions were shown in the random order and then again in

Six participants took part in the experiment. All participants signed the informed consent prior to

the experimental session and received monetary compensation. They had normal or corrected-to- 


\section{History computation}

The perceptual history that preceded each dominance phase duration was computed via leaky integrator with an exponential kernel as in [21]. The history was computed for two timescales, with the short timescale history $\tau=0.9 \cdot\left\langle T_{\text {dom }}>\right.$ and the long timescale $\tau=30 \cdot<T_{\text {dom }}>$, where $\left\langle T_{\text {dom }}>\right.$ is the mean dominance phase duration for a given participant, display, and contrast. We selected the short timescale time constant based on the results of [21] and so as to represent the most recent time history. Conversely, the purpose of the long timescale was to capture a possible slow drift in dominance phase durations [30]. Once the perceptual history was computed, we excluded the first two clear percepts of each block from the analysis as they did not have any preceding history for that state.

\section{Simulated data}

We have generated simulated data using a custom implementation of a spiking neural model of bistability [22] based on the code provided by Stepan Aleshin and Jochen Braun from Cognitive Biology group at Magdeburg University. The model was fitted individually for each participant and display from the first data set (Pastukhov et al., 2013b). We used a genetic algorithm [31] with the Kolmogorov-Smirnov test as a fitness function, to match the dominance phase distributions for the experimental and the simulated data as closely as possible. Note, however, that the fitness function did not include any information on the history dependence. The population size was 50 and the number of iterations 100 . After the final iteration, we used best model parameters to generate a time series with 1100 clear percepts (first 100 percepts were discarded to account for the initial "burn-in" period of the model), see Figure 3. Because the simulated data is stationary and does not exhibit any long-timescale drift, we computed perceptual history only for the short timescale $\left(\tau=0.9 \cdot<T_{\text {dom }}>\right)$. 


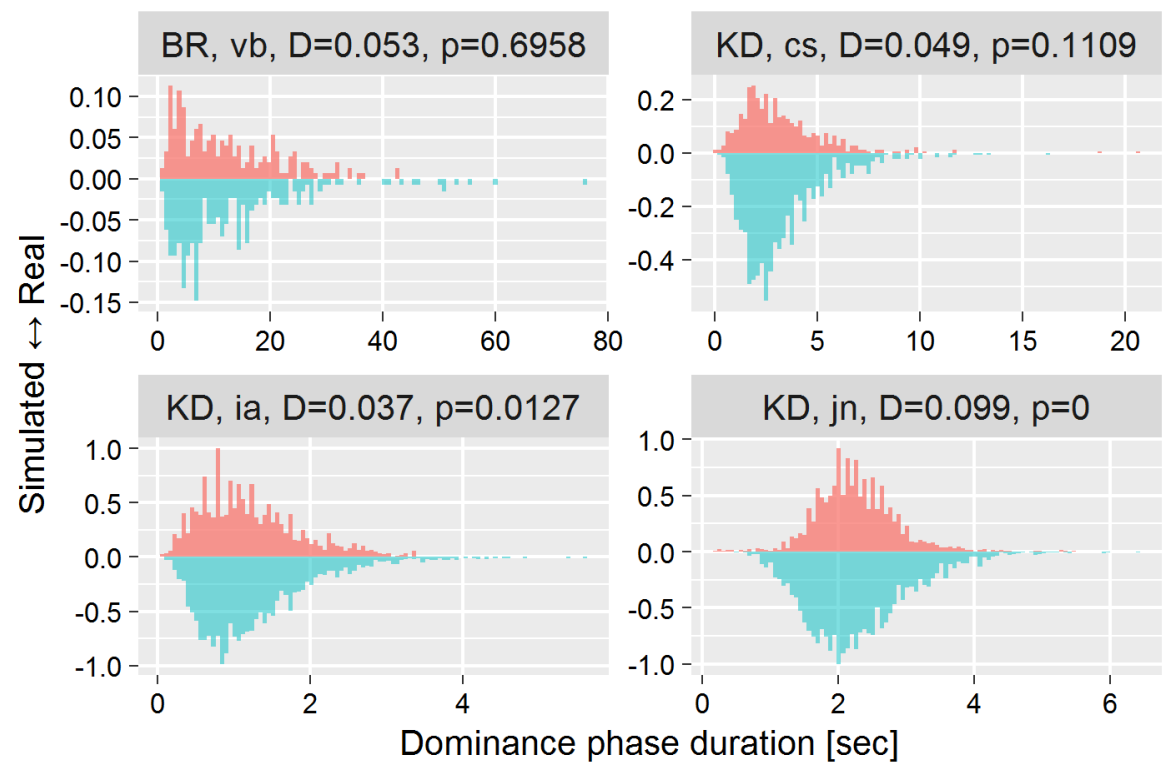

Figure 3. Example distributions of dominance phase durations for the experimental (upwards) and

matching simulated (downwards) data for four participants. We picked four participants based on the

Kolmogorov-Smirnov test $p$-value from worst-matched (BR, vb) to best-matched (KD, jn) with two

intermediate cases (at $1 / 3$ and 2/3). The title above each plot shows the display, participant code,

Kolmogorov-Smirnov test statistics and the $p$-value.

191

Model

We fitted each data set separately using a Bayesian hierarchical generalized linear model. Models for two parameters of gamma distribution - shape $(k)$ and scale $(\Theta)$ - were identical. For all groups, each parameter depended on prior perceptual history at two timescales, except for the simulated data, there they depended only on short-term history (see above). For contrasts and development data we also included the main effects of, correspondingly, contrast and age.

Before fitting the model, the data were centered as follows. Prior history was centered at its median value for each participant $\times$ timescale. For contrasts group, the contrast was centered at $50 \%$ value. For the age group, age was centered at a proposed mean young adult age of 21 years old. 


$$
\begin{aligned}
& \text { Duration }_{i} \sim \operatorname{Gamma}_{(}\left(k_{i}, \frac{1}{\theta_{i}}\right) \\
& \log \left(k_{i}\right)=\alpha_{p_{i}}+\beta_{\text {age }} \cdot \text { age }_{i}+\beta_{\text {contrast }} \cdot \text { contrast }_{i}+\sum_{t=1}^{2}\left(\beta_{p_{i}, t}\right) \cdot \text { History }_{i, t} \\
& \alpha \sim \operatorname{Normal}(0,10) \\
& \beta_{p, t} \sim \operatorname{Normal}\left(\mu_{t}^{\beta}, \sigma_{t}^{\beta}\right) \\
& \mu_{t}^{\beta} \sim \operatorname{Normal}\left(\mu^{\mu}, \sigma^{\mu}\right) \\
& \sigma_{t}^{\beta} \sim \operatorname{Normal}\left(\mu^{\sigma}, \sigma^{\sigma}\right) \\
& \mu^{\mu} \sim \operatorname{Normal}(0,1) \\
& \mu^{\sigma} \sim \operatorname{HalfCauchy}(0,10) \\
& \sigma^{\mu}, \sigma^{\sigma} \sim \operatorname{HalfCauchy}(0,1) \\
& \beta_{\text {age }}, \beta_{\text {contrast }} \sim \operatorname{Normal}(0,1)
\end{aligned}
$$

202 where $i$ is the row index, $t$ is the time scale index (1..2 range), $k_{i}$ is the shape parameter for the $i^{\text {th }}$

203 row, $p_{i}$ is the participant for the $i^{\text {th }}$ row, $a g e_{i}$ is participant's age, contrast ${ }_{i}$ is the display contrast, and History $y_{i, t}$ is the computed history value for $i^{\text {th }}$ row and $t^{\text {th }}$ time scale. The model for the scale parameter $\left(\Theta_{\mathrm{i}}\right)$ was identical.

The model was programmed and sampled using Stan probabilistic programming language [32].

[34]. The model was programmed and sampled using rstan [35]. The spiking neural model of bistability was implemented using Rcpp [36]. We used GA package [37] for genetic algorithm optimization.

Two out of three data sets (excluding the developmental data), the analysis code and the model code are available under Creative Commons Attribution 4.0 International Public License at 


\section{Results}

217 Our aim was to investigate how perceptual history affects the distribution of the following dominance phase durations. Specifically, we assumed that this distribution is well-approximated by Gamma (see Introduction for rationale) and we were interested in the history dependence of the two individual parameters, shape $(k)$ and scale $(\theta)$. To this end, for each dominance phase, we computed perceptual history for both short and long timescales. Then, we fitted each dataset using a hierarchical Bayesian model where both parameters linearly depended on both timescales. In addition, we included the effect of contrast and participants' age for, respectively, contrast and development data sets.

The shape and scale parameters of the fitted gamma distributions (without effects of history, contrast, and age) for all participants are plotted in Figure 4A. They show typical values with most shape parameters clustering between 2 and 4 and similarly tight clustering for the scale parameter. For the data set that used contrast manipulation, the effect of contrast matches that reported by van Ee [20]. Specifically, higher contrast led to a positive change for the shape parameter but a negative one for scale parameter (Figure 4B, left panel). However, we found no effect of age on either parameter of the gamma distribution (Figure 4B, right panel). 
A

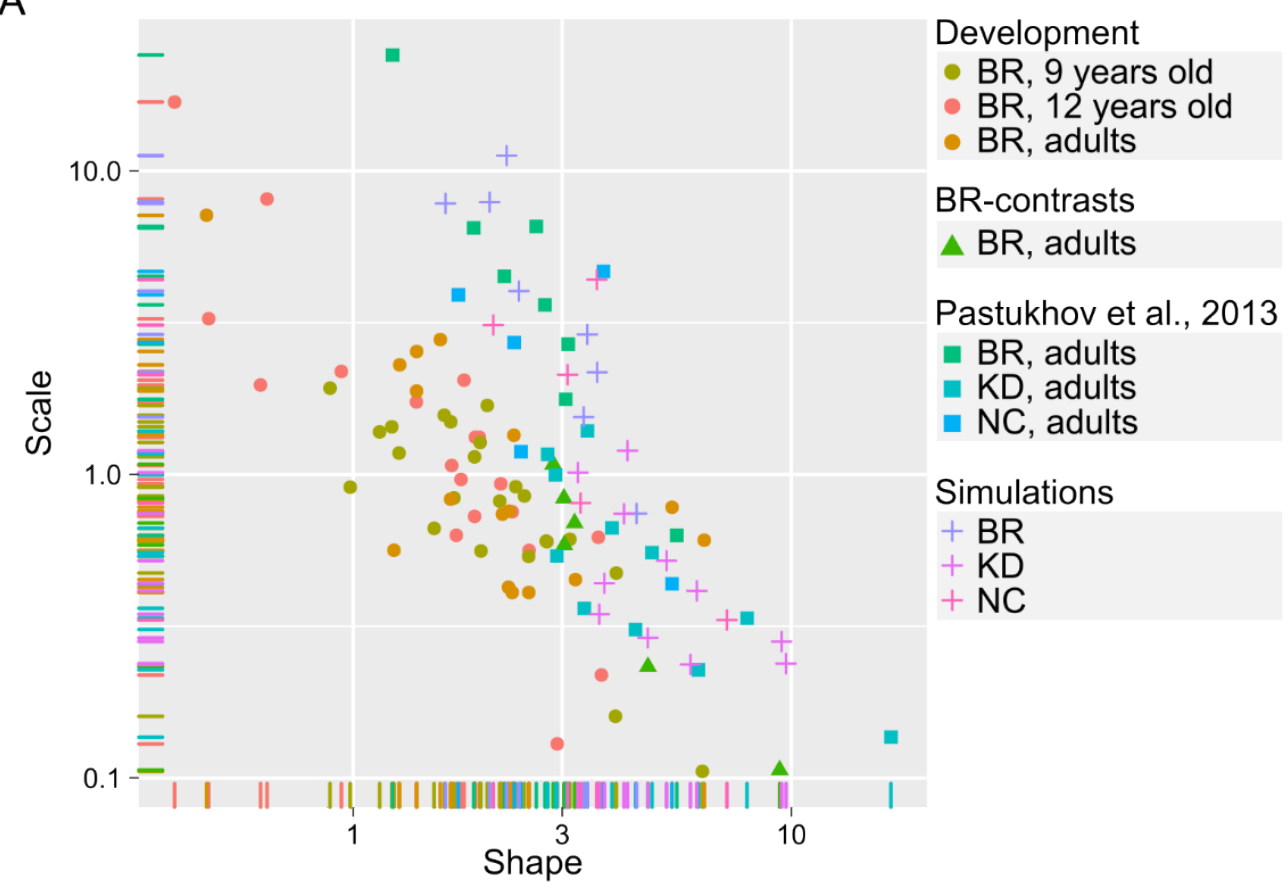

B

\section{Contrast}

B>0: $97.3 \%$

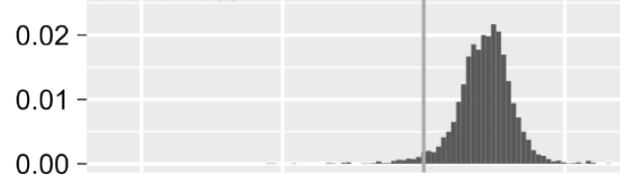

0.00

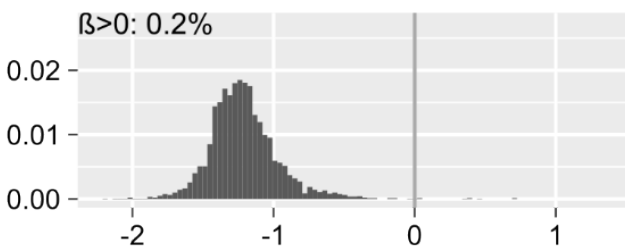

232

The history dependence of the parameters for both timescales is summarized in Figure 5. For the

241

Figure 4. Intercepts-only distribution parameters and main effects of contrast and age. A) The

scatterplot shows the intercepts-only parameters of the fitted gamma distribution for each

participant, including the simulated data. Shape and scale correspond to $\alpha^{\text {shape }}$ and $\alpha^{\text {shape }}$ except for development group data where it is equal to $\alpha^{\text {shape }}+\beta_{\text {age }}$ Age and $\alpha^{\text {scale }}+\beta_{\text {age }} \cdot$ Age (see the model for further details). B) Posterior distributions for main effects of contrast (Contrasts data set) and age (Development data set) for shape and scale parameters of the gamma distribution. Text inset in the top left corner shows the percentage of $\beta$-values above zero.

short timescale perceptual history, we found a consistent positive shift for the shape parameter 
242 across all five data sets, although the effect was weaker for Necker Cube (NC). For the scale

243 parameter, we also found a generally positive effect, although it was weaker and less consistent

244 across the data sets than that for the shape. The effect of the history for longer time scale was

245 more variable with mostly negative changes to both parameters indicating an overall speed-up

246 trend for perceptual alternations over time. 
bioRxiv preprint doi: https://doi org/10.1101/2020.08.06.239285; this version posted August $6,2020$. The copyright holder for this preprint (which was not certified by peer review) is the author/funder, who has granted bioRxiv a license to display the preprint in perpetuity. It is made available under aCC-BY 4.0 International license.
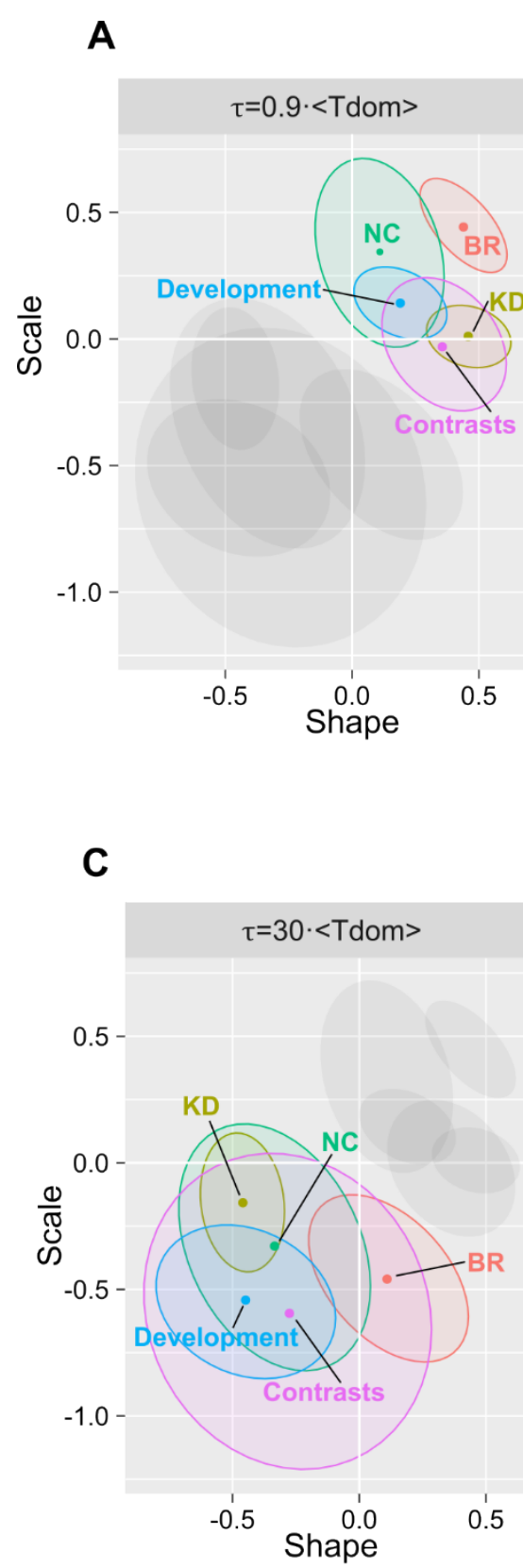
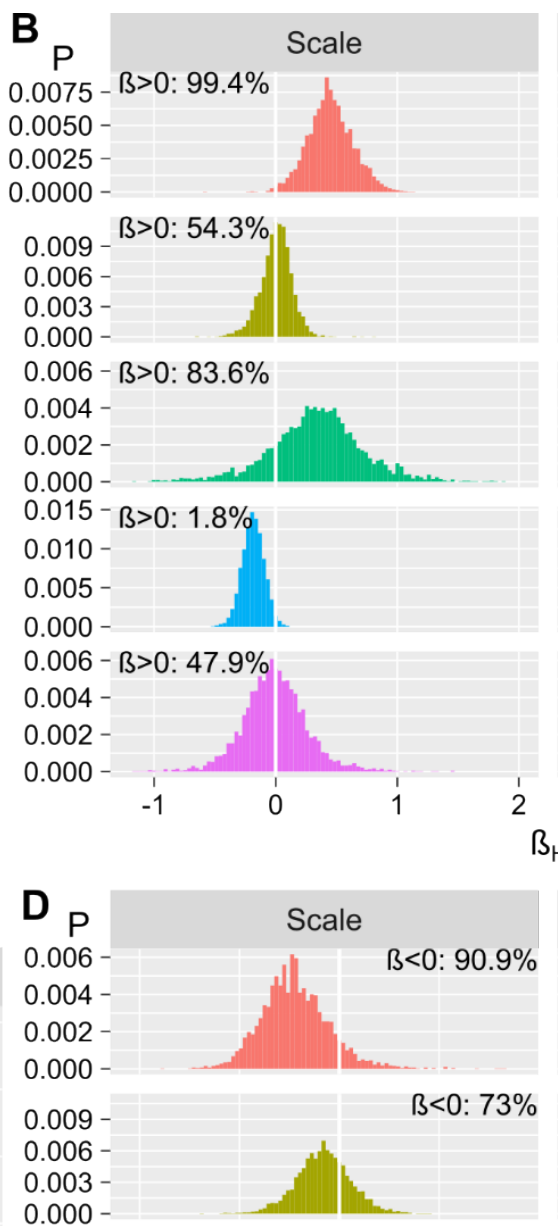

$0.000-$

$0.005-$

0.005
0.004
0.003
0.002

$0.002-$

0.000 -

$0.0075-$
$0.0050-$
$0.0025-$
$0.0000-$
$0.003-$
0.002
0.001
0.000

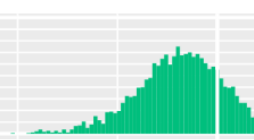

ß<0: $76.7 \%$

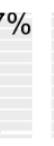

ß<0: $99.3 \%$
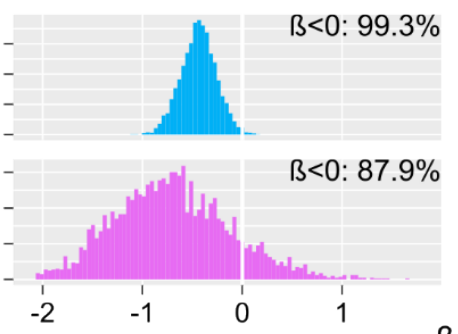

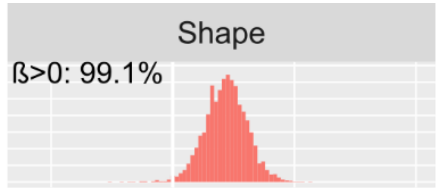

ß>0: $99.2 \%$
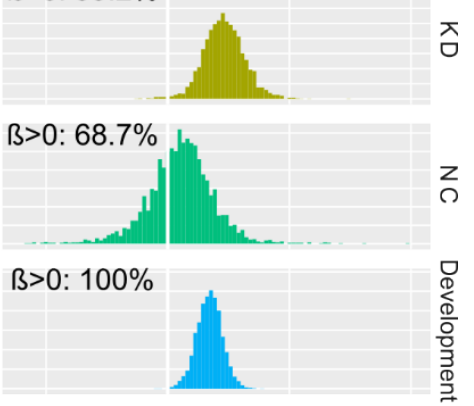

$ß>0: 95.4 \%$
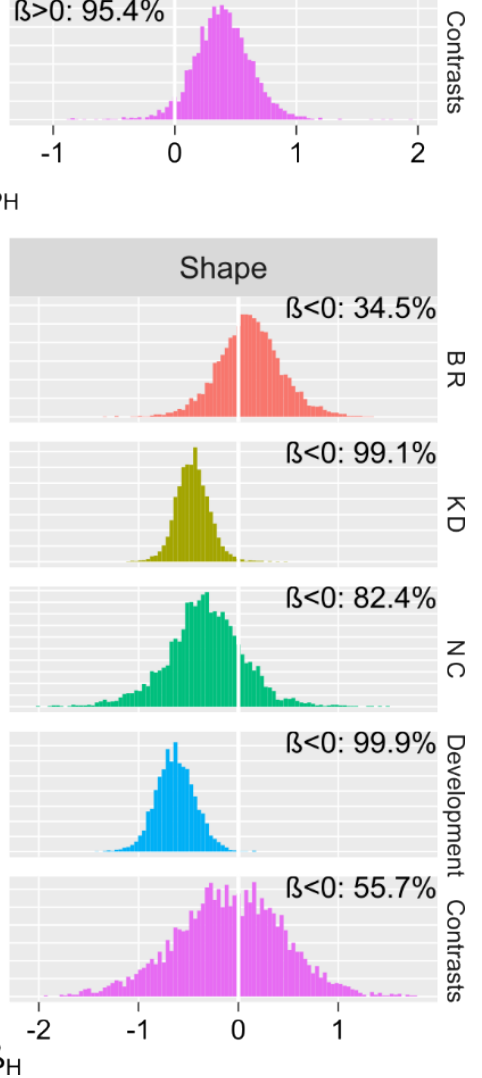

Figure 5. Posterior distributions for the main effect of prior history. A, B) short timescale $\left(\tau=0.9<T_{\text {dom }}>\right)$, 
We also compared the dominance phases of real observers with that generated by a spiking neural model of bistability [22]. The model was fit so as to match distributions of individual participants in three data sets (BR, KD, and NC) as closely as possible (see Methods for details). However, the

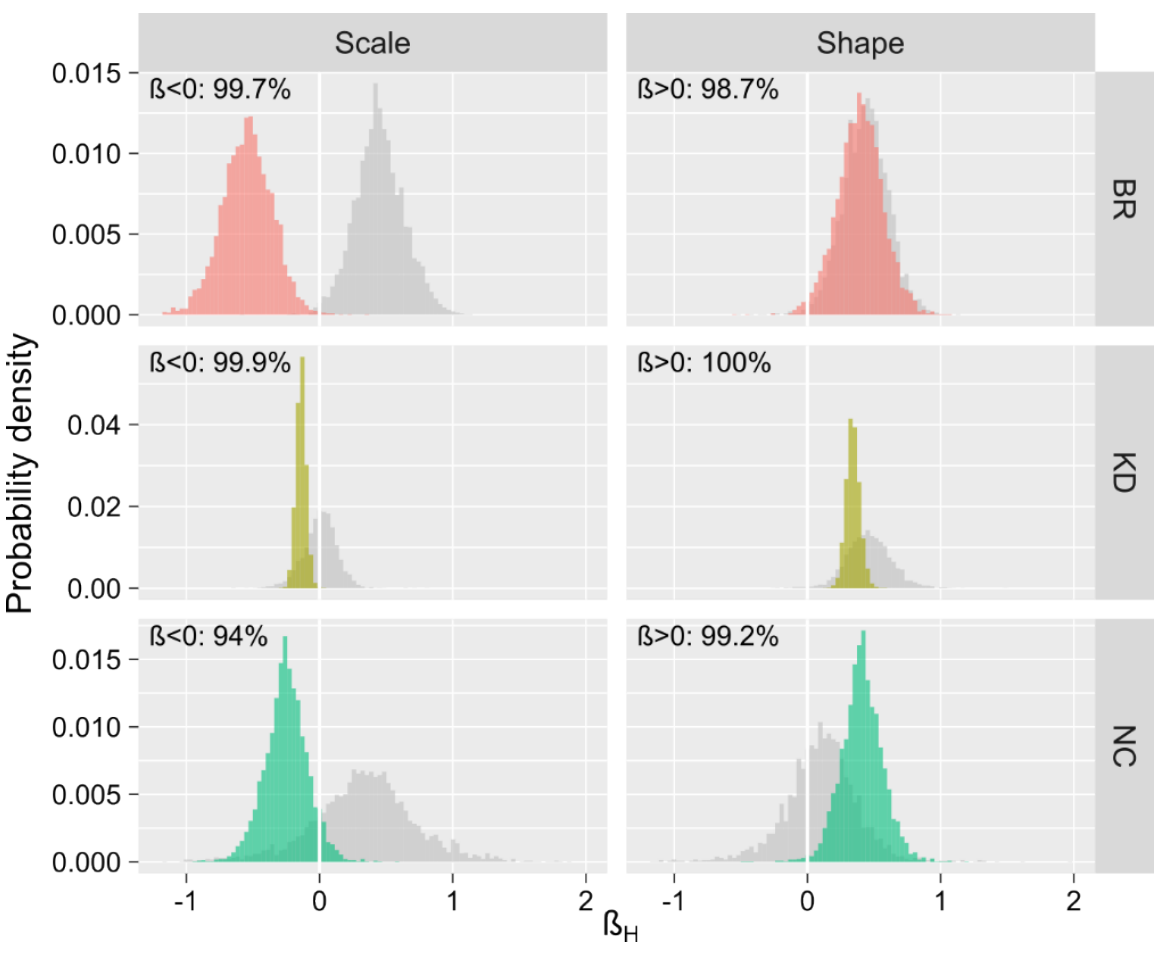




\section{Discussion}

The aim of this study was to characterize how prior perceptual history affects the distribution of the following dominance phase durations. To this end, we fitted the distributions of dominance phase durations using gamma distribution with its parameters being linearly dependent on prior perceptual history at two timescales, as well as contrast and age for two data sets. The two timescales were short ( $\tau=0.9<T_{\text {dom }}>$, where $<T_{\text {dom }}>$ is an average dominance phase duration) and long $\left(\tau=30<T_{\text {dom }}>\right)$, so as to capture both effect of the immediately preceding dominance state and the overall trend. For the former, we observed a consistent positive dependence for the shape parameter and a weaker, less consistent but also positive dependence for the scale parameter. For the latter, both parameters had a negative dependence on the longer-term prior history, indicating a general trend for faster perceptual alternations.

Of the two timescales, we focused on in the present study, the short one is often presumed to reflect the effect of perceptual adaptation $[20,21]$. As we explained in the introduction, perceptual adaptation is considered to be one of the two major drivers of perceptual alternations alongside the neural noise. This conceptualization predicts that predictability of the individual dominance phase duration depends on the balance between the two. When one of the competing populations is strongly adapted, the dominance duration tends to be longer reflecting the greater influence of adaptation. Conversely, when adaptation levels of two competing representations are comparable, the perceptual alternations and, therefore, dominance phase durations are determined primarily by noise $[21,26]$. Our results appear to bear out this prediction, as the higher levels of prior perceptual history - a proxy for perceptual adaptation - are associated with consistent positive changes for the shape parameters but only weaker and less consistent ones for the scale parameter of the gamma distribution. The overall effect is that the distribution is more normal-like and, therefore, is more regular when history/adaptation is high [25]. Conversely, it is more exponential-like when history/adaptation is low and perceptual switches are driven primarily 
by noise [27]. In short, our results tested and validated prior assumptions about the roles of noise and adaptation in multistable perception and provide further means of characterizing it.

The proposed analysis also provides a useful benchmark for models of multistability. Here, we used a spiking neural model of bistability [22] that is capable of capturing various properties of dominance phase distribution for continuous viewing [25]. Multiple more capable models are built on the same idea of mixing self-adaptation, cross-inhibition, and neural noise [38-40]. There exist a plethora of alternative approaches including stochastic accumulation in multiple assemblies [6], more subtle characterization of the time-series and, therefore, a more sensitive test for all these models. Importantly, this is the test that the model by Laing and Chow appears to fail. Although the model does exhibit the positive history-dependence of the shape parameter, it also shows a strong and consistent negative history-dependence for the scale parameter. These results do not completely match those of human participants, indicating that the model is incapable of fully capturing the underlying dynamics. We must note, however, that the model parameters were not optimized for history-dependence only for the overall match of the distribution shapes. product of the model itself. Therefore, it is possible that more thorough parameter-matching could produce a better match. Nonetheless, this discrepancy shows the usefulness of our approach for in-depth analysis of computational models of multistable perception. multistability. 
321

322

323

324

325

326

327

328

329

330

331

332

333

334

335

336

337

338

339

340

341

342

\section{Acknowledgments}

We thank Stepan Aleshin and Jochen Braun for providing us the code for the spiking neural model of bistability. I "thinking boy" image in Figure 1A is used under Public Domain license.

\section{References}

1. Carbon C-C. Understanding human perception by human-made illusions. Front Hum

Neurosci. 2014;8: 566. doi:10.3389/fnhum.2014.00566

2. Tong F, Meng M, Blake R. Neural bases of binocular rivalry. Trends Cogn Sci. 2006;10: 502-

11. doi:10.1016/j.tics.2006.09.003

3. Denham SL, Winkler I. The role of predictive models in the formation of auditory streams. J Physiol. 2006;100: 154-170. doi:10.1016/j.jphysparis.2006.09.012

4. Liaci E, Bach M, Van Elst LT, Heinrich SP, Kornmeier J. Ambiguity in tactile apparent motion perception. PLoS One. 2016;11: 1-22. doi:10.1371/journal.pone.0152736

5. Zhou W, Chen D. Binaral Rivalry between the Nostrils and in the Cortex. Curr Biol. 2009;19: 1561-1565. doi:10.1016/j.cub.2009.07.052

6. Cao R, Pastukhov A, Maurizio M, Braun J. Collective Activity of Many Bistable Assemblies Reproduces Characteristic Dynamics of Multistable Perception. J Neurosci. 2016;36: 6957-6972. doi:10.1523/JNEUROSCI.4626-15.2016

7. Leopold DA, Logothetis NK. Multistable phenomena: Changing views in perception. Trends Cogn Sci. 1999;3: 254-264. doi:10.1016/S1364-6613(99)01332-7

8. van Ee R. Dynamics of perceptual bi-stability for stereoscopic slant rivalry and a comparison with grating, house-face, and Necker cube rivalry. Vision Res. 2005;45: 29-40. doi:10.1016/j.visres.2004.07.039 
9. Levelt WJM. Note on the distribution of dominance times in binocular rivalry. Br J Psychol. 1967;58: 143-145. doi:10.1111/j.2044-8295.1967.tb01068.x

10. Cogan R. Distributions of durations of perception in the binocular rivalry of contours. J Gen

Psychol. 1973;89: 297-304. doi:10.1080/00221309.1973.9710832

11. Zhou YH, Gao JB, White KD, Merk I, Yao K. Perceptual dominance time distributions in

multistable visual perception. Biol Cybern. 2004;90: 256-263. doi:10.1007/s00422-004-0472-8

12. Lehky SR. Binocular rivalry is not chaotic. Proc R Soc London Ser B Biol Sci. 1995;259: 71-

76. doi:10.1098/rspb.1995.0011

13. de Marco A, Penengo P, Trabucco A. Stochastic models and fluctuations in reversal time of ambiguous figures. Perception. 1977;6: 645-656. doi:10.1068/p060645

14. Brascamp JW, van Ee R, Pestman WR, van den Berg A V. Distributions of alternation rates in various forms of bistable perception. J Vis. 2005;5: 1. doi:10.1167/5.4.1

15. Huguet G, Rinzel J, Hupe J. Noise and adaptation in multistable perception: Noise drives when to switch, adaptation determines percept choice. J Vis. 2014;14: 19-19. doi:10.1167/14.3.19 perceptual rivalry. Neuroreport. 2003;14: 1347-52. doi:10.1097/01.wnr.0000077553.91466.41 perception of visual ambiguous stimuli. Kybernetik. 1972;10: 139-144. Available: 
20. van Ee R. Stochastic variations in sensory awareness are driven by noisy neuronal

adaptation: evidence from serial correlations in perceptual bistability. J Opt Soc Am A. 2009;26:

21. Pastukhov A, Braun J. Cumulative history quantifies the role of neural adaptation in multistable perception. J Vis. 2011;11. doi:10.1167/11.10.12

22. Laing CR, Chow CC. A spiking neuron model for binocular rivalry. J Comput Neurosci.

23. Moreno-Bote R, Rinzel J, Rubin N. Noise-induced alternations in an attractor network model of perceptual bistability. J Neurophysiol. 2007;98: 1125-1139. doi:10.1152/jn.00116.2007

24. Noest AJ, van Ee R, Nijs MM, van Wezel RJA. Percept-choice sequences driven by interrupted ambiguous stimuli: a low-level neural model. J Vis. 2007;7: 10. doi:10.1167/7.8.10 perception balances stability and sensitivity. Front Comput Neurosci. 2013;7: 17. doi:10.3389/fncom.2013.00017

26. Brascamp JW, van Ee R, Noest AJ, Jacobs RHAH, van den Berg A V. The time course of binocular rivalry reveals a fundamental role of noise. J Vis. 2006;6: 1244-1256. doi:10.1167/6.11.8

27. Brascamp JW, Pearson J, Blake R, van den Berg A V. Intermittent ambiguous stimuli: Implicit memory causes periodic perceptual alternations. J Vis. 2009/09/18. 2009;9: 3-3. doi:10.1167/9.3.3 perception balances stability and sensitivity. Front Comput Neurosci. 2013;7: 17. 
29. Hudak M, Gervan P, Friedrich B, Pastukhov A, Braun J, Kovacs I. Increased readiness for adaptation and faster alternation rates under binocular rivalry in children. Front Hum Neurosci. 2011;5: 128. doi:10.3389/fnhum.2011.00128

30. Mamassian P, Goutcher R. Temporal dynamics in bistable perception. J Vis. 2005;5: 361375. doi:10.1167/5.4.7

31. Scrucca L. GA: A Package for Genetic Algorithms in R. J Stat Softw. 2013;53. doi:10.18637/jss.v053.i04

32. Carpenter B, Gelman A, Hoffman MD, Lee D, Goodrich B, Betancourt M, et al. Stan: A Probabilistic Programming Language. J Stat Softw. 2017;76. doi:10.18637/jss.v076.i01

33. R Core Team. R: A Language and Environment for Statistical Computing. Vienna, Austria; 2019. Available: https://www.r-project.org/

34. Wickham H, Averick M, Bryan J, Chang W, McGowan L, François R, et al. Welcome to the Tidyverse. J Open Source Softw. 2019;4: 1686. doi:10.21105/joss.01686

35. Carpenter B, Gelman A, Hoffman MD, Lee D, Goodrich B, Betancourt M, et al. Stan: A Probabilistic Programming Language. J Stat Softw. 2017;76. doi:10.18637/jss.v076.i01

36. Eddelbuettel D, François R. Rcpp: Seamless R and C++ Integration. J Stat Softw. 2011;40. doi:10.18637/jss.v040.i08

37. Scrucca L. GA: A Package for Genetic Algorithms in R. J Stat Softw. 2013;53. doi:10.18637/jss.v053.i04

38. Noest AJ, van Wezel RJ a. Dynamics of temporally interleaved percept-choice sequences: interaction via adaptation in shared neural populations. J Comput Neurosci. 2012;32: 177-95. doi:10.1007/s10827-011-0347-7 
410 39. Wilson HR. Minimal physiological conditions for binocular rivalry and rivalry memory.

411 Vision Res. 2007;47: 2741-2750. doi:10.1016/j.visres.2007.07.007

412 40. Shpiro A, Moreno-Bote R, Rubin N, Rinzel J. Balance between noise and adaptation in

413 competition models of perceptual bistability. J Comput Neurosci. 2009/01/07. 2009;27: 37-54.

414 doi:10.1007/s10827-008-0125-3

415 41. Moreno-Bote R, Knill DC, Pouget A. Bayesian sampling in visual perception. Proc Natl Acad

Sci U S A. 2011;108: 1-6. doi:10.1073/pnas.1101430108

417 42. Weilnhammer V, Stuke H, Hesselmann G, Sterzer P, Schmack K. A predictive coding account

418 of bistable perception - a model-based fMRI study. Daunizeau J, editor. PLOS Comput Biol.

419 2017;13: e1005536. doi:10.1371/journal.pcbi.1005536

420 43. Atmanspacher H, Filk T, Romer H. Quantum Zeno features of bistable perception. Biol

421 Cybern. 2004;90: 33-40. doi:10.1007/s00422-003-0436-4 